\title{
Culturally tailored DSMES: A new key for South Asian patients with Type 2 Diabetes Mellitus
}

\author{
Hongxiu Luo ${ }^{1}$, Annah Kuriakose ${ }^{2}$, Geethika Thota ${ }^{3}$, Naveen Mehrotra*2 \\ ${ }^{1}$ Division of Endocrinology, Nutrition, and Metabolism, Department of Medicine, Saint Peter's University Hospital, NJ, USA \\ ${ }^{2}$ SKN South Asian Diabetes Center, Department of Medicine, Saint Peter's University Hospital, NJ, USA \\ ${ }^{3}$ Department of Medicine, Saint Peter's University Hospital, NJ, USA
}

Received: September 13, 2021

DOI: $10.5430 /$ dcc.v8n 4 p14
Accepted: November 27, $2021 \quad$ Online Published: December 15, 2021

URL: https://doi.org/10.5430/dcc.v8n4p14

\begin{abstract}
South Asians have an exceptionally high risk for developing Type 2 diabetes mellitus. It is very challenging for healthcare providers to successfully manage diabetes and control glucose levels at target due to the unique lifestyle of the South Asian population. Culturally tailored diabetes self-management education and support (DSMES) can be more effective in guiding South Asian patients with Type 2 diabetes. Unique considerations to address lifestyle modification for South Asians include a diet that typically consists of a high carbohydrate to lipids/proteins ratio, preference for high glucose index fruits, regular intake of traditional sweets or desserts, late afternoon tea break followed by late dinner, lack of vigorous exercise (yoga or walking being the preferred activity), lack of DSMES knowledge and skills, and poor access to culturally appropriate resources for diabetes care. We present a 38-year-old male diagnosed with diabetes four years ago who showed poor glucose control before our intervention. Our interventions included education on the importance of blood glucose monitoring, exercise, and diet. Based on our experience with this case, we propose the following recommendations for a tailored approach to DSMES for South Asian patients with Type 2 diabetes: make appropriate dietary changes (decrease total daily caloric intake, decrease the percentage of carbohydrates, add low glucose index fruits and vegetables, avoid late afternoon tea breaks, eat dinner before $8 \mathrm{PM}$ ); incorporate appropriate daily physical activity; and monitor blood glucose daily for prompt feedback.
\end{abstract}

Key Words: Culturally, South Asian, Lifestyle, Diabetes, Self-management, Exercise, DSMES, Diet

\section{INTRODUCTION}

South Asians (persons from India, Pakistan, Bangladesh, Nepal, Sri Lanka, Afghanistan, Bhutan, or the Maldives) have an exceptionally high risk for developing Type 2 diabetes mellitus. ${ }^{[1]}$ There is a high prevalence of Type 2 diabetes among the South Asian diaspora in the West, including in the United States and Canada. It is very challenging for healthcare providers to successfully prevent the development of Type 2 diabetes and ideally control glucose levels at target among these populations because of the heterogeneity of ethnic diversity, geography, religions, languages, beliefs, dietary patterns, and eating habits. Diabetes self-management education and support [DSMES], led by board-certified endocrinologists and board-certified diabetes educators, is designed to support informed decision-making, self-care behaviors, problem-solving, active patient participation, and collaboration with the healthcare team. ${ }^{[2,3]}$ It is well known as a clinically meaningful and statistically significant tool to im-

\footnotetext{
*Correspondence: Naveen Mehrotra; Email: nmehrotra@sknfoundation.org; Address: SKN South Asian Diabetes Center, Department of Medicine, Saint Peter's University Hospital, NJ, USA.
} 
prove glycated hemoglobin [HbA1C] levels, reduce the risk of diabetes-related complications, and reduce the number of hospitalizations for the general population and persons from diverse cultural and ethnic groups. ${ }^{[3]}$ However, reports show that most $(75 \%)$ DSMES is ineffective in reducing HbA1C levels among the migrant South Asian population, even after native language translation. ${ }^{[4]}$ We believe that a more personalized or culturally tailored DSMES could be appropriate and effective in guiding South Asian patients with Type 2 diabetes. In this case report, we share a classic case to demonstrate the significant efficacy of a culturally tailored DSMES on Type 2 diabetes control through specific lifestyle changes.

\section{Case PResentation}

A 38-year-old male, originally from Andhra Pradesh, India, was referred by his primary care provider [PCP] to the endocrinologist for Type 2 diabetes control. He was diagnosed with diabetes four years before this visit and was prescribed sitagliptin/metformin 50/1,000 mg PO daily at the time. Unfortunately, his glucose control worsened despite medication over the past four years, with an HbA1C increase from $6.8 \%$ to $8.6 \%$. In his first visit with the endocrinologist, he received culturally tailored DSMES, as well as a medication dose change (sitagliptin/metformin 50/1,000 mg PO bid). By the second visit three months later, his $\mathrm{HbA} 1 \mathrm{C}$ had decreased from $8.5 \%$ to $6.4 \%$, and he had lost more than $5 \%$ of his body weight (from $183 \mathrm{lbs}$ to $173 \mathrm{lbs}$ ).

\subsection{Our approach}

\subsubsection{Changes to diet}

The most significant changes in the patient's lifestyle came through diet. Notable changes included adding one serving of both fruit and daily vegetables (either as a raw green smoothie or baked greens before dinner). In addition, the patient began to exercise portion control with carbohydrates: he cut out one serving of rice daily and decreased portion sizes for rice/chapati and dessert. There was no change in non-vegetarian consumption (fish and chicken). Finally, the patient switched his tea from Indian tea with milk to green tea without milk or sweeteners. There was no significant difference in timing for meals and snacks other than adding one serving of fruit in the afternoon.

\subsubsection{Changes to exercise}

Before the intervention, the patient did not do any regular exercise. Afterward, the patient added one hour of guided yoga every morning before any food intake.

\subsubsection{Changes to blood glucose monitoring}

Before the intervention, the patient did not monitor blood glucose. Afterward, he began to monitor daily, using it as a feedback system. He currently monitors 2-3 times per week, either fasting or two hours after dinner.

Studies showed that sitagliptin/metformin 100/2,000 mg per day lowered HbA1C by about $2.07 \%^{[5]}$ in a group with $\mathrm{HbA} 1 \mathrm{C}$ in the range of $8 \%-9 \%$. Still, this intervention had not worked with our patient before adapting specific lifestyle interventions. In our case, the patient successfully controlled his $\mathrm{HbA} 1 \mathrm{C}$ to target levels and lost 10 pounds of body weight in three months due to lifestyle changes and antihyperglycemic medication adherence.

\section{Discussion}

Why was one-time DSMES so effective for this patient to control his diabetes, notably when previous interventions had failed? The key lies in the culturally tailored lifestyle modification for this unique case:

\subsection{Diet}

(1) Carbohydrate intake. South Asian meals typically include a very high carbohydrate to lipids/proteins ratio. This is because a relatively high percentage of South Asians are vegetarian, some of whom also avoid animal protein/fat-based food items and eggs. Nonvegetarians may only eat fish, chicken, or goat a few times per week. Given these limitations, South Asians tend to take in more carbohydrates to compensate for caloric requirements.

(2) Deficit of raw vegetables and fresh fruits. Anecdotally, we have noted that most South Asian diets may have a relative deficit of vegetables and fresh fruits in daily meals. For those who eat fruits regularly, high glucose index fruits are preferred, like mangos and bananas.

(3) Desserts. In addition, many South Asians eat traditional sweets or desserts regularly. ${ }^{[6]}$

\subsection{Lifestyle}

(1) Late dinner. Anecdotally, many South Asian Americans/immigrants have a habit of a late afternoon tea break (5-6PM) and subsequently late dinner. The main component of the tea break is often pure carbohydrates, like cookies, cakes, or other traditional sweets, which postpones dinnertime due to satiety from carbohydrate intake. In addition, a late dinnertime (after $8 \mathrm{PM}$ ) is a significant risk factor for Type 2 diabetes in South Asian populations. ${ }^{[7]}$

(2) Lack of exercise. Compared to their Western counterparts, South Asians have a more sedentary lifestyle. ${ }^{[8]}$ They rarely use gyms; more gentle activities like yoga or walks in the neighborhood are more common for those who exercise. These, however, may not be suf- 
ficient for weight loss or glucose control. In addition, seniors (age $>65$ ), who have a higher prevalence of Type 2 diabetes, may have lower motivation for exercise.

\subsection{Recommendations}

Based on our experience with this case, we propose the following recommendations for South Asian patients with diabetes for the appropriate use of DSMES:

(1) Refer South Asian patients with diabetes to endocrinology or DSMES programs at the time of diagnosis of diabetes $(\mathrm{HbA} 1 \mathrm{C}>6.5 \%)$ or even prediabetes $(5.7 \%$ $6.5 \%$ ). In addition, the clinical training of PCPs should be strengthened in diabetes care, as diabetes is one of the most common diseases they encounter.

(2) Recommend the following dietary changes:

- Limit total caloric intake by eliminating traditional sweets and cut portion sizes by calorie counting based on South Asian food menus. ${ }^{[9]}$
- Decrease the percentage of carbohydrates in the whole meal regimen to less than $40 \%-50 \%$. In addition, increase low glucose index vegetables and fruits in the diet.

- Avoid late afternoon tea breaks with pure carbohydrates. If this is not possible, switch to fruits/vegetables.

- Avoid dinner later than 8 PM.

(3) Recommend daily exercise. Traditional home exercises like yoga and dance should be done for at least 60 minutes/day. Post-prandial walking for 30-60 minutes, 2-3 times a day, is also strongly recommended.

(4) Recommend daily blood glucose monitoring, emphasizing post-prandial fingersticks (two hours after a big meal).

\section{CONFlicts OF INTEREST Disclosure}

The authors declare they have no conflicts of interest.

\section{REFERENCES}

[1] Mohan V. Why are Indians more prone to diabetes? J Assoc Physicians India. 2004; 52: 468-474.

[2] Haas L, Maryniuk M, Beck J, et al. National standards for diabetes self-management education and support. Diabetes Care. 2013; 36 Suppl 1(Suppl 1): S100-S108. PMid:23264420. https://doi .or g/10.2337/dc13-S100

[3] Powers MA, Bardsley J, Cypress M, et al. Diabetes Self-management Education and Support in Type 2 Diabetes: A Joint Position Statement of the American Diabetes Association, the American Association of Diabetes Educators, and the Academy of Nutrition and Dietetics. Diabetes Care. 2015; 38(7): 1372-1382. PMid:26048904. https://doi.org/10.2337/dc15-0730

[4] Navodia N, Wahoush O, Tang T, et al. Culturally Tailored SelfManagement Interventions for South Asians With Type 2 Diabetes: A Systematic Review. Can J Diabetes. 2019; 43(6): 445-452. PMid:31375180. https://doi.org/10.1016/j.jcjd.2019.04 .010

[5] Goldstein BJ, Feinglos MN, Lunceford JK, et al. Effect of initial combination therapy with sitagliptin, a dipeptidyl peptidase- 4 inhibitor, and metformin on glycemic control in patients with Type2 diabetes published correction appears in Diabetes Care. 2007; 30(8): 19791987. PMid:17485570. https://doi .org/10.2337/dc07-0627

[6] Priya G, Grewal E, Kalra S. Sweet Cravings in Diabetes: Desserts that are not so Sinful. J Pak Med Assoc. 2019; 69(4): 595-597.

[7] Tang TS, Halani K, Sohal Pet al. Do Cultural and Psychosocial Factors Contribute to Type 2 Diabetes Risk? A Look Into Vancouver's South Asian Community. Can J Diabetes. 2020; 44(1): 14-21. PMid:31444060. https://doi.org/10.1016/j.jcjd.2019.04 .015

[8] Afaq S, Kooner AS, Loh M, et al. Contribution of lower physical activity levels to higher risk of insulin resistance and associated metabolic disturbances in South Asians compared to Europeans. PLoS One. 2019; 14(5): e0216354. PMid:31063476. https : //doi.org/10.1371/journal.pone.0216354

[9] Mian SI, Brauer PM. Dietary education tools for South Asians with diabetes. Can J Diet Pract Res. 2009; 70(1): 28-35. PMid:19261204. https://doi.org/10.3148/70.1.2009.28 